\title{
一种新型三氮唑化合物的光物理性质及其与血清白蛋白的键合研究
}

\author{
何文英 ${ }^{a}$ 司宏宗 ${ }^{b}$ 栾峰 ${ }^{c}$ 吴禄勇 ${ }^{a}$ \\ 周纪龙 ${ }^{a}$ 何明霞 ${ }^{a}$ 陈光英*, $a$ \\ ( ${ }^{a}$ 海南师范大学化学化工学院 海口 571158) \\ ( ${ }^{b}$ 青岛大学计算科学与工程技术研究中心 青岛 266071) \\ ( ${ }^{c}$ 烟台大学化学化工学院 烟台 264005)
}

\begin{abstract}
摘要 1-(1-萗基)-5-苯基-1 $H$-1,2,3-三㟇(1-(naphthalen-1-yl)-5-phenyl-1 $H$-1,2,3-triazole, 简称 NPTA)是一种新合成的三氮 唑化合物, 研究了其光谱特征及其生物活性. 利用 PM3 半经验方法的 Polak-Ribiere 共轭梯度法得到了优化的 NPTA 分 子结构, 进而用分子对接显示与人血清白蛋白(HSA)的键合模式及位点，再利用紫外光谱、多种苂光光谱法等技术表征 了 NPTA 及其键合 HSA 的光谱性质. 结果表明: 共轭多烯 $\pi$ 键的存在使得 NPTA 呈现特征的苂光与紫外光谱. 分子模 拟的结果表明 NPTA 可以嵌于 HSA 分子的疏水腔内, 并与精氨酸 Arg222 形成四个氢键; 位点竞争实验确定了 NPTA 在 HSA 亚结构域的位点 II 位发生作用. 二维及三维荧光光谱显示 NPTA 可以猝灭 HSA 的内源苂光，使其构象发生变 化; 紫外、同步荧光扫描证实 NPTA 主要猝灭了 HSA 色氨酸残基的荧光, 并影响了 HSA 的微环境; 较小的各向异性值 说明 NPTA 与 HSA 结合后生成的配合物弛豫时间较短, 结合的较松. 苂光滴定法求得不同温度下 (299, 309 和 319 K) NPTA 与 HSA 作用的键合常数, 键合模式为典型的疏水作用, 与分子模拟的结果相一致. 另外, 测定了 NPTA 与键合 HSA 相关的几种物理化学参数.
\end{abstract}

关键词 NPTA; HSA; 光谱法; 键合作用

\section{Photophysical Behavior and the Binding to Human Serum Albumin of a Novel Triazole Compound}

\author{
He, Wenying ${ }^{a} \quad$ Si, Hongzong ${ }^{b} \quad$ Luan, Feng $^{c} \quad$ Wu, Luyong ${ }^{a}$ \\ Zhou, Jilong $^{a} \quad$ He, Mingxia ${ }^{a} \quad$ Chen, Guangying*, \\ ( ${ }^{a}$ College of Chemistry and Chemical Engineering, Hainan Normal University, Haikou 571158, China) \\ ( ${ }^{b}$ Institute for Computational Science and Engineering, Qingdao University, Qingdao 266071, China) \\ ( ${ }^{c}$ College of Chemistry and Chemical Engineering, Yantai University, Yantai 264005, China)
}

Abstract 1-(Naphthalen-1-yl)-5-phenyl-1H-1,2,3-triazole (NPTA), a novel synthesized triazole compound has been characterized for its photophysical properties and bioactivity. The structure of NPTA was optimized by semi-empirical PM3 method using the Polak-Ribiere algorithm. Molecular modeling was furtherly performed to reveal the binding mode and site to human serum albumin (HSA). The spectroscopic properties and the binding to HSA of NPTA were investigated by absorption spectra, synchronous fluorescence, 3D fluorescence spectra and fluorescence polarization. The results indicated that the characteristic absorption and fluorescence spectrum could be attributed to the conjugated polyene $\pi$ bond of NPTA. Molecular docking showed NPTA moiety bound to the hydrophobic cavity of HSA and there are four hydrogen bonds interactions between NPTA and the residues Arg222. Fluorescent displacement measurements confirmed that NPTA bound HSA on site II. The 2D and 3D fluorescence spectroscopes of NPTA-HSA system indicated that NPTA quenched strongly the intrinsic fluorescence of HSA and induced a conformational change of the protein. The absorption and synchronous fluorescence spectra showed that NPTA could quench the fluorescence of tryptophan mainly and have effect on the microenvironment around HSA in aqueous solution. The low anisotropy values suggested that NPTA molecules were bound in a motionally unrestricted environment introduced by HSA. The binding constants $\left(10^{4}\right.$ magnitude) and the number of binding sites $(n \approx 1)$ between NPTA and HSA at different temperatures (299, 309 and $319 \mathrm{~K})$ were calculated from relevant fluorescence titration data, which indicated the strong binding between NPTA and HSA. Meanwhile, from the thermodynamic parameter calculation, it could be shown that the acting force was mainly the hydrophobic interactions, which was in good agreement with molecular modeling studies. Under the conditions studied, the values of the negative charge density $(\delta)$, the dissociation constants $\left(K_{\mathrm{d}}\right)$ and quantum yield $(\Phi)$ of NPTA-HSA system were calculated.

Keywords NPTA; human serum albumin (HSA); spectroscopy; binding

*E-mail: chgying123@163.com

Received December 16, 2012; published March 8, 2013.

Project supported by the National Natural Science Foundation of China (No. 21162009).

项目受国家自然科学基金(No. 21162009)资助. 


\section{1 引言}

1,2,3-三氮唑类化合物是一类重要的杂环化合物, 大量研究表明, 该类化合物具有独特的化学性质, 在有 机化学、有机金属化学、药物化学和材料化学等诸多领 域具有广阔的应用前景. 尤其在医学方面的应用, 如 $\beta$ 内酞胺酶的抑制作用, 抗 HIV, 抗菌, 抗过敏, 抗肿瘤, 抗病毒, 抗癫㾁, 止痛以及控制与心血管疾病有关的钾 的代谢平衡等性质 ${ }^{[1]}$. 另外, 生物活性测试表明, 三氮 唑化合物具有较强的杀菌和一定的调节植物生长活性 的能力 ${ }^{[2]}$. 1-(1-菜基)-5-苯基-1H-1,2,3-三唑(1-(naphthalen-1-yl)-5-phenyl-1H-1,2,3-triazole, 简称 NPTA，结构 见图 1)是新合成的三氮唑类化合物 ${ }^{[1]}$, 作为一种潜在开 发利用的药物小分子, 研究它的光物理性质及其与蛋白 质的相互作用及所引起的氨基酸、构象的变化 ${ }^{[3]}$, 对于 设计此类新药、笁选药物等都具有指导意义. 本文利用 荧光光谱、紫外光谱法及量子力学表征了 NPTA 的物理 化学性质; 再结合同步荧光、荧光偏振、三维荧光及分 子模拟研究了 NPTA 与 HSA 的相互作用, 确定了键合 参数及作用力模式, 定性分析了 NPTA 对 HSA 二级结 构及微环境的影响.

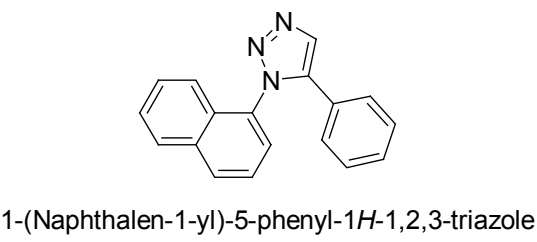

图 1 NPTA 的化学结构

Figure 1 The structure of NPTA

\section{2 结果与讨论}

\subsection{NPTA 分子的优化结构及光物理性质}

利用 HyperChem 软件包, 获得优化的 NPTA 的分 子几何构型(见图 2). 从图 2 可看出: NPTA 分子没有显 示出平面构型, 只是其中的萮环处于同一平面, 这与文 中后面研究它与 HSA 的分子模拟得出的结果一致. 这 说明: NPTA 分子的空间位阻较大, 可形成三维的非平 面分子结构. 通过量子化学计算的 NPTA 稳定构像能量 为 $-3950.47 \mathrm{kcal} / \mathrm{mol}$, 最高占有轨道能量 (HOMO) 为 $-9.30 \mathrm{eV}$, 最低末占轨道能量 (LUMO) 为 $-1.00 \mathrm{eV}$.

图 3 为 $\mathrm{NPTA}$ 在甲醇溶液 $\left(1.3 \times 10^{-3} \mathrm{~mol} \cdot \mathrm{L}^{-1}\right)$ 中的 紫外-可见光谱 $(A)$ 与苂光光谱(B). 从图 3(A)可以看出, NPTA 的吸收带主要位于 200 到 $300 \mathrm{~nm}$ 之间, 这个吸收 带是由单键桥联的菜环与三氮唑及苯环形成的整体共 轭骨架的 $\pi$ - $\pi$ *跃迁导致的. 其最大吸收峰位于 $222 \mathrm{~nm}$, 推测应是萗环的 E1 特征吸收带(位于 $220 \mathrm{~nm}$ ), 由于 NPTA 分子中三唑环 1-位氮原子的给电子能力, 使得 荟环的 E1 特征吸收发生轻微红移 $2 \mathrm{~nm}$. 在 $209 \mathrm{~nm}$ 处出 现一肩峰, 可能是杂环化合物母体的特征吸收带 ${ }^{[4]}$.

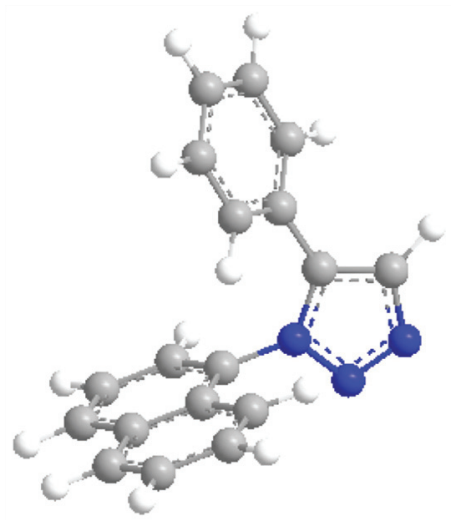

图 2 用 Polak-Ribiere 共轭梯度法优化得到的 NPTA 分子结构 Figure 2 The structure of NPTA optimized using the Polak-Ribiere algorithm

图 3(B)中的曲线 a 为激发波长为 $321 \mathrm{~nm}$ 时, NPTA 的荧光发射光谱, 显示最大发射峰位置在 $381 \mathrm{~nm}$ 处, 并 在 $647 \mathrm{~nm}$ 处有一小峰出现; 而曲线 $\mathrm{b}$ (红色标注)为发射 波长为 $381 \mathrm{~nm}$ 时, NPTA 的荧光激发光谱, 显示在约 $312 \mathrm{~nm}$ 处表现出一个轻微的精细结构的苂光发射峰. 以上两个荧光光谱图表明, 在 $10^{-3} \mathrm{~mol} \cdot \mathrm{L}^{-1}$ 的数量级浓 度条件下, NPTA 有较强的苂光强度(达 8000 左右), 这 是由于 NPTA 不仅具有大的共轭 $\pi$ 键结构, 也存在 $N$ 的 给电子取代基的原因 ${ }^{[5]}$. 推测 NPTA 应有较大的苂光量 子效率, 这与前面的量子化学结果是相一致的, 暗示 NPTA 可作为一种良好的发光材料, 有待于下一步深入 研究.

\subsection{NPTA 与 HSA 作用的分子模拟}

血清白蛋白是血浆中最为丰富的蛋白质, 含有多种 可配位基团, 能与生物体内许多内源或外源物质作用. 尤其是研究 NPTA 这种新型化合物与 HSA 的相互作用, 有利于我们深入认识它在生物体系中所发挥的重要功 能. HSA 分子由三个结构域组成, 每个结构域又分别含 有 A, B 两个亚结构域, 以相对的槽口方式形成圆筒状 结构, 这种特殊的结构构成了一疏水腔, 在腔内不仅包 埋了几乎所有疏水性氨基酸残基，也是小分子键合 HSA 的主要区域 ${ }^{[6]}$.

图 4 及 5 为 NPTA 键合 HSA 的分子模拟图 $(8 \AA$ 范 围内的氨基酸残基). 无论从棍状模型的图 4 或飘带模 型的图 5 均可看出, 整个 NPTA 分子可以键合在 HSA 分子的疏水腔内, 且很靠近 HSA 分子的苯丙氨酸 Phe211 和色氨酸 Trp 214, 进一步说明 NPTA 与 HSA 的 键合存在疏水作用. 另外, 显示出 NPTA 分子中的苯 环、荎环及单键桥联的三氮唑环并未共平面, 且存在与 精氨酸 Arg222 结合的四个氢键, 键长分别是 2.582 , 2.612, 2.498 和 $2.082 \AA$. 得到相应的吉布斯自由能 $\Delta G$ 值为 $-21.73 \mathrm{~kJ} \cdot \mathrm{mol}^{-1}$, 与实验数据较接近 $(\Delta G=$ $\left.-23.12 \mathrm{~kJ} \cdot \mathrm{mol}^{-1}\right)$. 

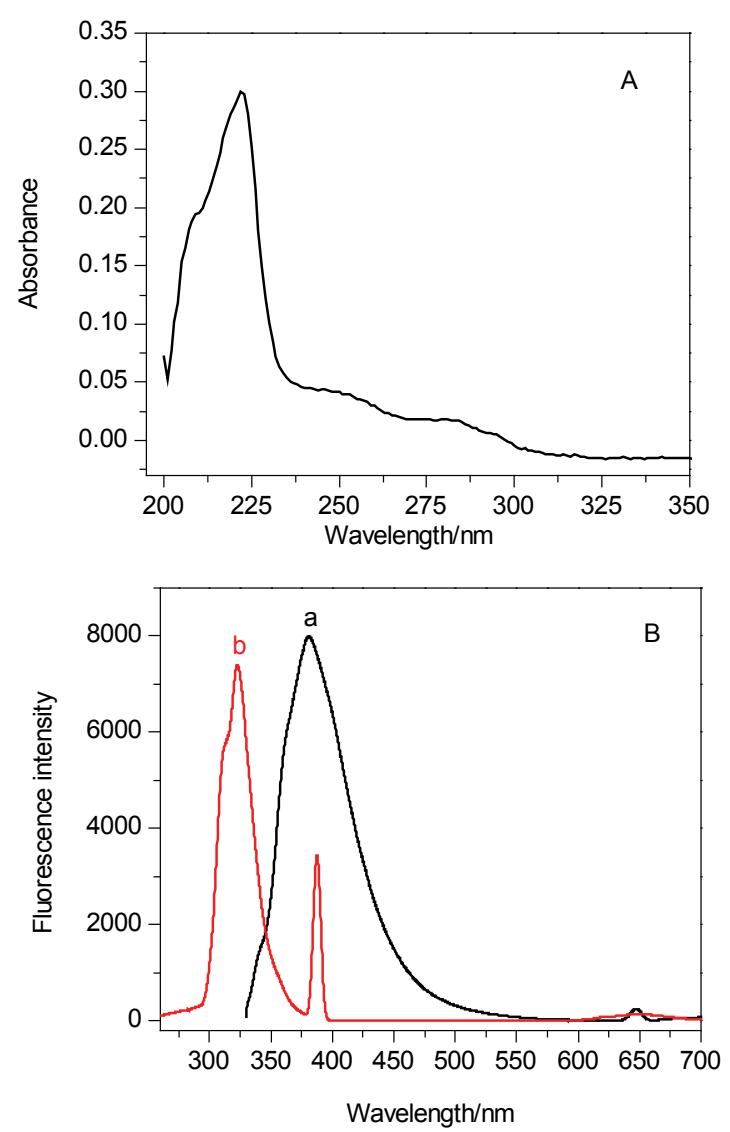

图 3 NPTA 在甲醇溶液中的紫外光谱(A)及苂光光谱图(B) (a 为发射 光谱, $\mathrm{b}$ 为激发光谱)

Figure 3 The absorbance spectra (A) and fluorescence spectra (B) in methanol (a: emission spectra, b: excitation spectra). $c_{\mathrm{NPTA}}=1.3 \times 10^{-3}$ $\mathrm{mol} \cdot \mathrm{L}^{-1}$

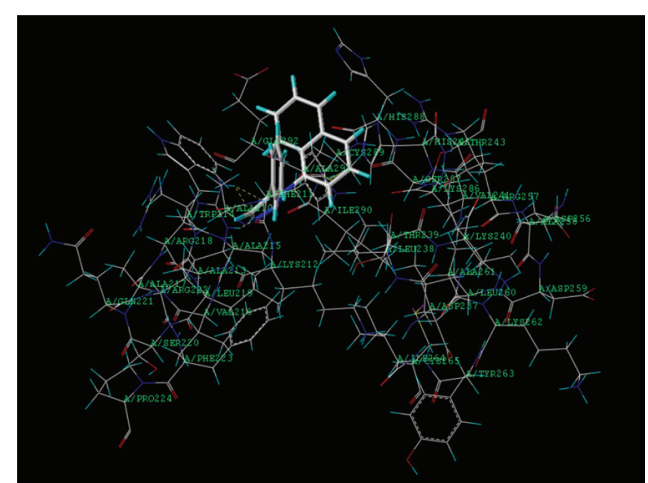

图 4 NPTA 与 HSA 的棍状模型对接图

Figure 4 The binding mode between NPTA and HSA, only residues around $8 \AA$ of NPTA is displayed. The residues of HSA are represented using line and the NPTA structure is represented using ball and stick model

\subsection{NPTA 与 HSA 作用的光谱表征}

荧光光谱法是一种研究蛋白质分子构象的有效方 法, 能提供许多物理参数, 从不同角度反映分子的成键 和结构情况. 在蛋白质分子中, Trp 残基的苂光强度最 大, 对微环境的变化很敏感, 常作为内源荧光探针来研

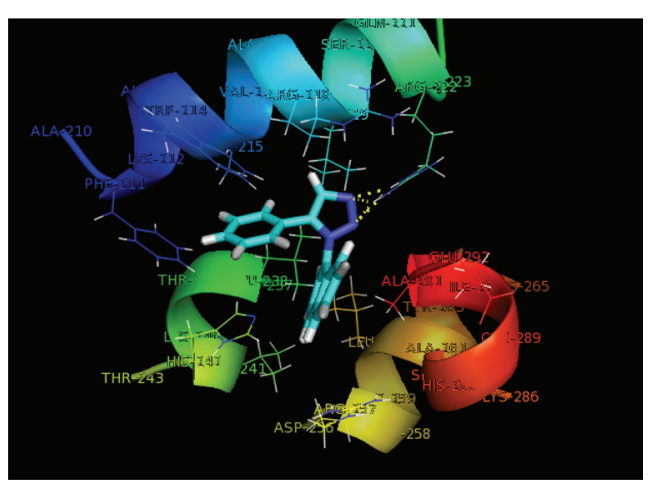

图 5 NPTA 与 HSA 的飘带状模型对接图

Figure 5 The zonal binding mode between NPTA and HSA only residues around $8 \AA$ of NPTA is displayed. The residues of HSA are represented using zonal and the NPTA structure is represented using ball and stick model. The hydrogen bond between NPTA and HSA is represented using dashed line

究溶液状态下蛋白质的构象. 而 HSA 在约 $210 \mathrm{~nm}$ 的最 大吸收峰反映了其 $\alpha$-螺旋结构的信息 ${ }^{[7]}$. 图 6 为固定 HSA 浓度, 变化 NPTA 浓度时得到的苂光光谱(A)与紫 外光谱(B)图. 图 6(A)显示：随着 NPTA 浓度的加大, 相比不加 NPTA 时的 HSA(图 6(A)中曲线 1), HSA-NPTA 体系的苂光强度逐渐减小 (图 6(A)中曲线 $2 \rightarrow 9$ ), 即 NPTA 猝灭了 HSA 的内源色氨酸残基的荧光(忽略 NPTA 的荧光强度). 从谱图及形状看: 峰型较规则, 并 伴随有红移现象 $(348 \rightarrow 352 \mathrm{~nm})$. 这可能归结为两个原 因: NPTA 的分子结构中存在的 $\mathrm{N}$ 原子为给电子基团, 导致荧光光谱产生位移，及吸收光强度的增大(见图 6(B)); 再是由于加入 NPTA后，使得 HSA 的微环境极性 增大, 从而形成规则的发射光谱 ${ }^{[8]}$.

图 6(B)为固定 HSA 浓度, 变化 NPTA 浓度时得到 的紫外光谱图. 可看出: 随 NPTA 浓度的增加, 发生最 大吸收峰位置红移现象 $(214 \rightarrow 220 \mathrm{~nm})$. 这进一步说明 NPTA 结构中有较大的共轭体系, 并使得 HSA 的 $\alpha$-螺旋 结构发生一定的变化.

在同一测定条件下，进行了三维荧光光谱的测定 (图 7), 考察在 NPTA 存在时, HSA 溶液构象的变化. 从 图 7 中可见, NPTA 猝灭了 HSA 的苂光, 但相比 HSA 在 Tris 缓冲溶液中的三维荧光谱图(a图), 加入 NPTA(此时 浓度为 HSA 的 11 倍左右)后, 药物-蛋白体系苂光峰的 形状发生了较大的变化, 峰型变得不规则( $\mathrm{b}$ 图), 这在图 6 中的二维荧光谱图中并没有表现, 可能是因为形成了 三唑氮原子与菜环的 $\mathrm{p}-\pi$ 共轭体系，导致在不同的激发 波长处有不同的激发方式，从而形成不同的发射光谱图 形, 但总的变化趋势是由不太规则渐渐变化为规则均匀 的图形; 另一方面, 从三维图谱的结果可以清晰的看到 NPTA 猝灭 HSA 的内源苂光时, 体系的最大激发与发射 波长的变化(图中红色标注), 推测 NPTA 与 HSA 的键合 作用使 HSA 的构象有一定的变化. 

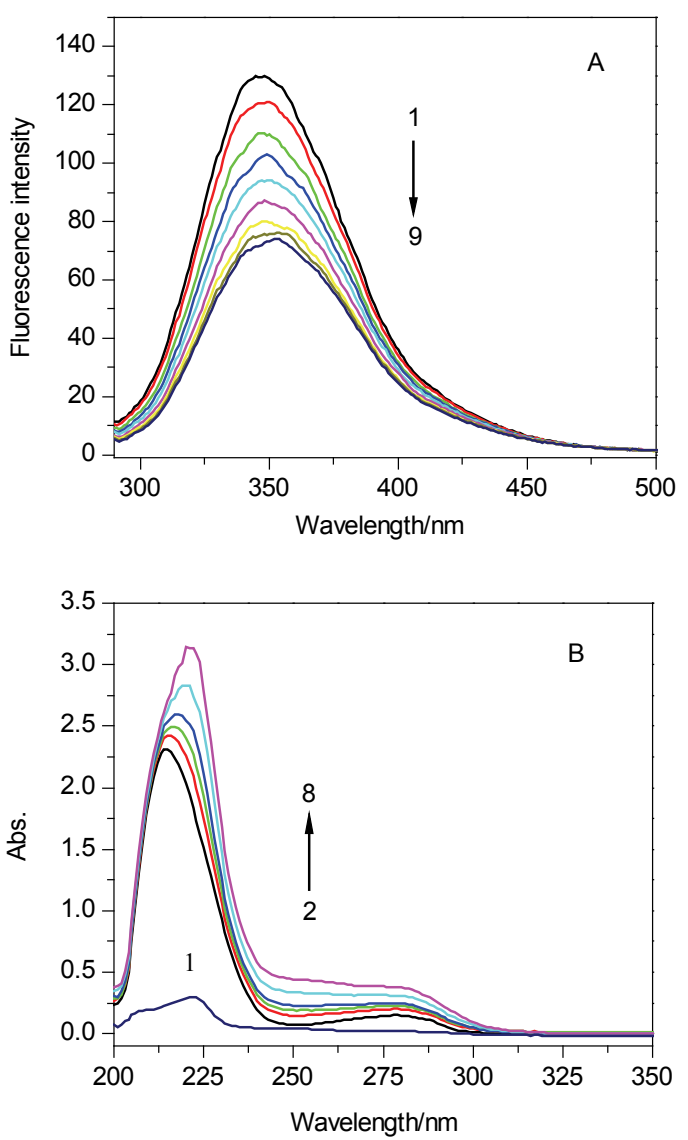

图 6 NPTA-HSA 体系荧光光谱(A)与紫外光谱图(B)

Figure 6 (A) The fluorescence spectra of NPTA-HSA system. The concentration of HSA was $3.0 \times 10^{-6} \mathrm{~mol} / \mathrm{L}$ while the NPTA concentration corresponding to $0,3.33,6.67,10,16.67,23.33,30,36.67,43.33$ $\left(\times 10^{-6}\right) \mathrm{mol} \cdot \mathrm{L}^{-1}$ from 1 to $9 . \lambda_{\mathrm{ex}}=285 \mathrm{~nm}, T=298 \mathrm{~K}$; (B) UV absorption spectra of NPTA-HSA, 1: [NPTA] $=4.33 \times 10^{-6} \mathrm{~mol} \cdot \mathrm{L}^{-1}, 2 \sim 8$ $\left([\mathrm{NPTA}] \times 10^{-6} \mathrm{~mol} \cdot \mathrm{L}^{-1}\right): 0,3.33,10,16.67,23.33,30,36.67,43.33 \times$ $10^{-6} \mathrm{~mol} \cdot \mathrm{L}^{-1}, T=298 \mathrm{~K}$

图 7a 中 HSA 的三维荧光光谱的光谱峰 $\left(\lambda_{\mathrm{ex}} / \lambda_{\mathrm{em}}\right)$ 为 $280 / 350 \mathrm{~nm}$, Stokes 位移为 $70 \mathrm{~nm}$, 图 $7 \mathrm{~b}$ 中 NPTA-HSA 体系的三维荧光光谱的光谱峰 $\left(\lambda_{\mathrm{ex}} / \lambda_{\mathrm{em}}\right)$ 为 $280 / 365 \mathrm{~nm}$, Stokes 位移为 $85 \mathrm{~nm}$. 可以看出两者的峰位置和峰形有 一定的差别, 由于蛋白质主要表现为色氨酸残基的苂 光, 图 7a 表明 HSA 中色氨酸残基的 $\lambda_{\mathrm{em}}(350 \mathrm{~nm})$ 较短, Stokes 位移较小 $(70 \mathrm{~nm})$, 而加入 NPTA 后 HSA 中色氨 酸残基的 $\lambda_{\mathrm{em}}(365 \mathrm{~nm})$ 较长, Stokes 位移较大 $(85 \mathrm{~nm})$, 即 NPTA 使 HSA 所处微环境的极性增强，同时荧光强度明 显降低. 这说明了 NPTA 的存在对 HSA 的色氨酸残基 所处的微环境引起的差异, 也表明了色氨酸残基所处的 HSA 分子中构象的变化.

为更加明确 NPTA 对 HSA 中 Trp 残基的影响, 采用 固定波长的同步扫描法. 通常 $\Delta \lambda=15 \mathrm{~nm}$ 对应的同步荧 光光谱显示酪氨酸残基 $T y r$ 的光谱特征，而 $\Delta \lambda=60 \mathrm{~nm}$ 的则呈现的是 Trp 残基的光谱特征. 图 8 为固定 HSA 浓 度，依次增大 NPTA 的浓度所测得的同步荧光光谱图，

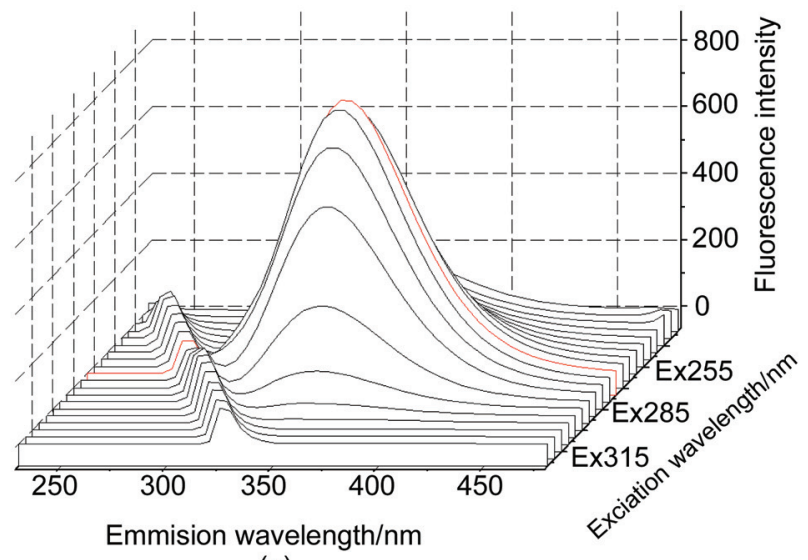

(a)

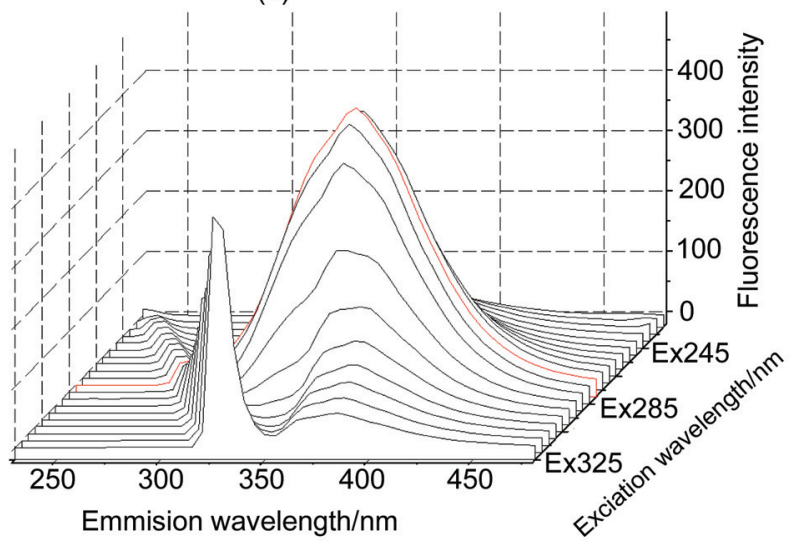

(b)

图 $7 \mathrm{HSA}$ 的三维荧光光谱图 $\left(c_{\mathrm{HSA}}=3.0 \times 10^{-6} \mathrm{~mol} \cdot \mathrm{L}^{-1}\right)$ (a) 和 NPTA-HSA 体系的三维荧光光谱图 $\left(c_{\mathrm{NPTA}}=3.9 \times 10^{-5} \mathrm{~mol} \cdot \mathrm{L}^{-1}\right)(\mathrm{b})$

Figure 7 The $3 \mathrm{D}$ fluorescence spectra of HSA (a) $\left(c_{\mathrm{HSA}}=3.0 \times 10^{-6}\right.$ $\left.\mathrm{mol} \cdot \mathrm{L}^{-1}\right)$ and the 3D fluorescence spectra of NPTA-HSA system (b) $\left(c_{\mathrm{NPTA}}=3.9 \times 10^{-5} \mathrm{~mol} \cdot \mathrm{L}^{-1}\right)$

从图中可看出: NPTA 的存在, 同时猝灭了 HSA 的 Trp 和 Tyr 残基的荧光. 不同之处是: NPTA 猝灭 Trp 残基的 程度较大(荧光强度从 150 降至 70 左右), 并且体系发生 红移(339 $\rightarrow 344 \mathrm{~nm}$ ); 而 NPTA 猝灭 Tyr 残基的程度较小 (荧光强度从 25 降至 7.5 左右), 其最大荧光发射峰位置 基本未变. 以上结果说明 NPTA 对 Trp 残基的影响较大, 使其所处的微环境疏水性降低, 亲水性增大.

另外，荧光偏振法是研究溶液中分子间相互作用的 一种有效而灵敏的方法，通过苂光偏振或各向异性值的 变化, 可了解药物与蛋白结合的一些信息. 图 9 为同一 温度的条件下, 改变 NPTA 的浓度而测得的 HSA 的各 向异性值. 可看出, 随着药物浓度比的增大 $(0 \rightarrow 53.33 \times$ $\left.10^{-6} \mathrm{~mol} \cdot \mathrm{L}^{-1}\right)$, 各向异性值较小, 均在 $0.023 \sim 0.029$ 之 间, 说明 NPTA 与 HSA 结合后生成的配合物弛豫时间 较短, 结合的较松, 或者说是 NPTA 的加入, 使 HSA 在 伸展从螺旋到无规卷曲变化时, 由于挠性增大, 所以偏 振较小.

\subsection{NPTA 与 HSA 作用的键合参数}

本文基于苂光猝灭法来确定 NPTA 与 HSA 作用的 

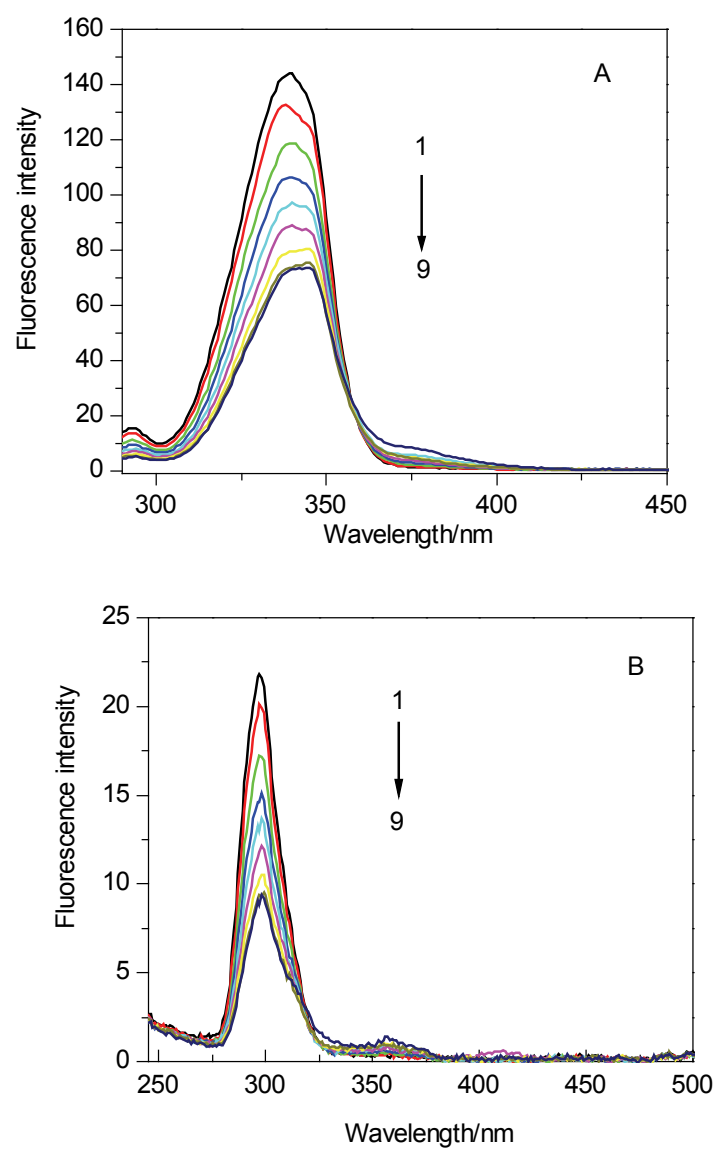

图 8 NPTA-HSA 体系的同步苂光光谱图(A: $\Delta \lambda=60 \mathrm{~nm}, \mathrm{~B}: \Delta \lambda=15$ $\mathrm{nm})$

Figure 8 Synchronous fluorescence spectra of NPTA-HSA system. (A: $\Delta \lambda=60 \mathrm{~nm}, \mathrm{~B}: \Delta \lambda=15 \mathrm{~nm}) .1:[\mathrm{HSA}]=3.0 \times 10^{-6} \mathrm{~mol} \cdot \mathrm{L}^{-1} ; 2 \sim 9$ $\left([\mathrm{NPTA}] \times 10^{-6} \mathrm{~mol} \cdot \mathrm{L}^{-1}\right): 3.33,6.67,10,16.67,23.33,30,36.67,43.33$

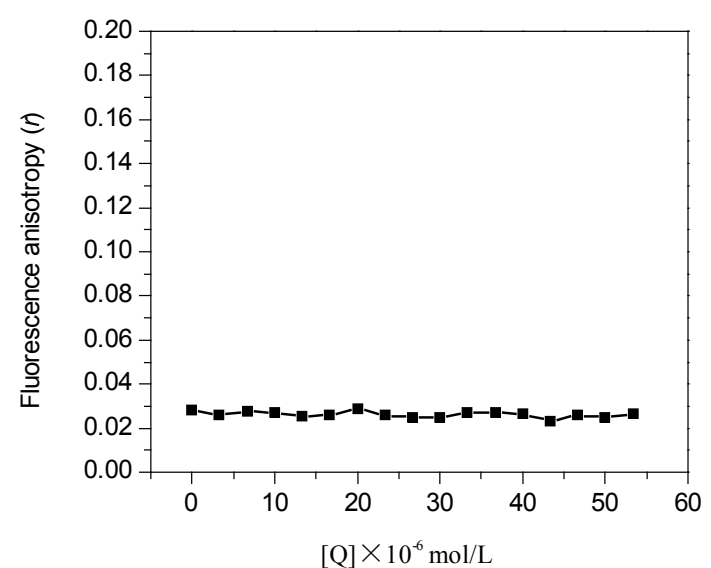

图 9 HSA 的各向异性值(变化 NPTA 的浓度)

Figure 9 Variation in the fluorescence anisotropy $(r)$ of HSA (3.0X $\left.10^{-6} \mathrm{~mol} \cdot \mathrm{L}^{-1}\right)$ with increasing NPTA concentration $\left(\lambda_{\mathrm{ex}}=285 \mathrm{~nm}, \lambda_{\mathrm{em}}=\right.$ $348 \mathrm{~nm})$.

各个键合参数, 包括键合常数、键合位点数、键合距离、 键合作用力形式及键合部位.

选定三个不同温度 $(299,309$ 及 $319 \mathrm{~K})$ 进行苂光强 度的测定, 依据 Scatchard 公式求取键合常数及键合位
点数 ${ }^{[9]}$ (图 10). 得到的键合常数与键合位点数见表 1 , 可 看出 NPTA 与蛋白质的键合常数较大，且随温度的增 大，键合位点数呈减小趋势. 说明 NPTA 对 HSA 的键合 作用较强，且温度的变化会影响键合常数的大小.

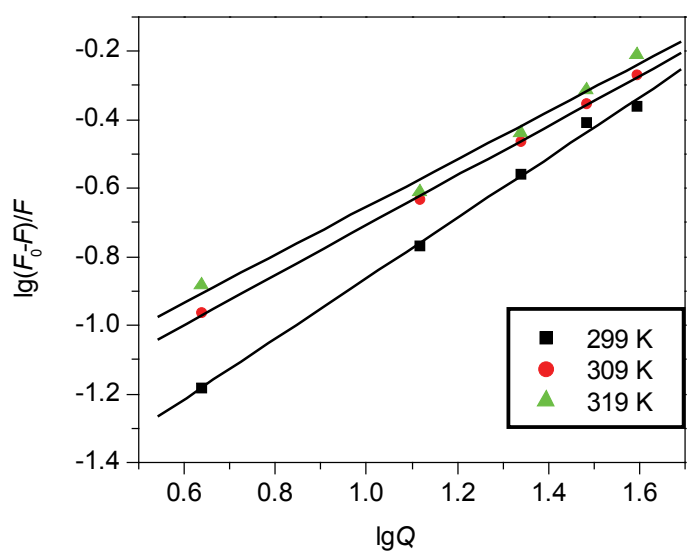

图 10 NPTA-HSA 体系的 Scatchard 图

Figure 10 The Scatchard plot for NPTA-HSA at $\mathrm{pH} 7.40$ $[\mathrm{HSA}]=3.0 \times 10^{-6} \mathrm{~mol} / \mathrm{L}$. pH $7.40 ; \lambda_{\mathrm{ex}}=285 \mathrm{~nm}, \lambda_{\mathrm{em}}=348 \mathrm{~nm}$

表 1 不同温度下 NPTA-HSA 体系的键合参数及热力学常数

Table 1 The binding parameters and thermodynamic parameters of NPTA-HSA interaction at $\mathrm{pH} 7.40$

\begin{tabular}{|c|c|c|c|c|c|}
\hline $\begin{array}{c}\text { Temp./ } \\
\mathrm{K}\end{array}$ & $\begin{array}{c}K / \\
\left(\times 10^{4} \mathrm{~mol}^{-1} \cdot \mathrm{L}\right)\end{array}$ & $n \quad r / \mathrm{nm}$ & $\begin{array}{c}\Delta G / \\
\left(\mathrm{kJ} \cdot \mathrm{mol}^{-1}\right)\end{array}$ & $\begin{array}{c}\Delta S / \\
\left(\mathrm{J} \cdot \mathrm{mol}^{-1} \cdot \mathrm{K}^{-1}\right)\end{array}$ & $\begin{array}{c}\Delta H / \\
\left(\mathrm{kJ} \cdot \mathrm{mol}^{-1}\right)\end{array}$ \\
\hline 299 & 1.794 & 0.88 & -23.121 & & \\
\hline 309 & 3.677 & 0.735 .22 & -24.190 & 106.880 & 8.836 \\
\hline 319 & 4.443 & 0.70 & -25.259 & & \\
\hline
\end{tabular}

利用 Förster 能量转移理论确定 NPTA 对 HSA 的键 合距离 $r$ 值, 由此推测 NPTA 与 HSA 结合的空间部 位 ${ }^{[10]}$. 将 HSA 看做能量转移给体, NPTA 为能量转移受 体, 测定 HSA 的荧光光谱(a)与 NPTA 的吸收光谱(b)重 叠图(图 11). 再依据相关公式 ${ }^{[11]}$, 求得光谱重叠部分的 积分面积 $J$ 值为 $16.50611 \times 10^{-14} \mathrm{~cm}^{3} \cdot \mathrm{L} \cdot \mathrm{mol}^{-1}$, 临界距 离 $R_{0}$ 为 $3.28 \mathrm{~nm}, r$ 值为 $5.22 \mathrm{~nm}$. 计算结果表明: 在 NPTA 与 HSA 之间确实发生了非辐射能量转移; 同时 NPTA 在 HSA 的结合位置与 Trp 残基之间的距离 $r$ 值小 于 $7 \mathrm{~nm}$, 这也与前面的分子模拟结果相一致.

利用 Van't Hoff 方程，根据不同温度下结合常数的 值可求得 NPTA 与 HSA 作用的热力学常数(表 1), 依此 判断其主要作用力类型 ${ }^{[12]}$. 正的 $\Delta S$ 及 $\Delta H$ 值表明 NPTA 主要以疏水作用键合 HSA, 负 $\Delta G$ 值则表明这种键合过 程是自发进行的. 这也与文中前面的分子模拟的结果相 一致, 再次证明了 NPTA 键合在 HSA 亚结构域的疏水 腔. 综合热力学计算与分子模拟的结果, 得出: NPTA 主 要以疏水作用及氢键键合 HSA.

为确定 NPTA 在 HSA 的空间键合区域, 本实验选 择对 H S A 具有特异结合性的三种竞争试剂 (phenylbutazone (PB)、flufenamic acid (FA) 和 digitoxin 


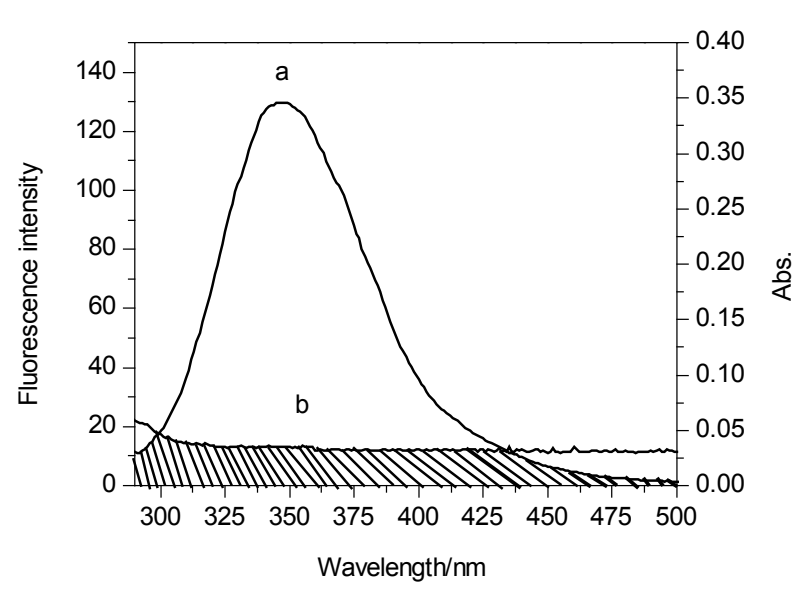

图 11 HSA 的苂光光谱(a)与 NPTA 的吸收光谱(b)重叠图 Figure 11 Overlapping between the fluorescence emission spectrum of HSA (a) $\left(\lambda_{\mathrm{ex}}=285 \mathrm{~nm}\right)$ and absorption UV spectrum of NPTA (b). $c_{\mathrm{NPTA}} / c_{\mathrm{HSA}}=1: 1$.

(Dig)分别作为位点 I、位点 II、位点 III 位的标记药物 ${ }^{[13]}$, 研究它们与 NPTA 对 HSA 的竞争结合情况. 在分别含 一定浓度三种竞争试剂的存在条件下, 测定不同 NPTA-HSA 体系的苂光强度, 用相关公式处理所得的 数据, 与不加竞争试剂时体系的键合常数相比 $(1.794 \times$ $\left.10^{4} \mathrm{~mol}^{-1} \cdot \mathrm{L}\right)$, 发现键合常数均发生了变化, 分别为: $3.277 \times 10^{4} \mathrm{~mol}^{-1} \cdot \mathrm{L}(\mathrm{PB}), 3.792 \times 10^{4} \mathrm{~mol}^{-1} \cdot \mathrm{L}(\mathrm{FA})$, $1.295 \times 10^{4} \mathrm{~mol}^{-1} \cdot \mathrm{L}(\mathrm{Dig})$. 其中, FA 竞争 NPTA 的效果 较明显, 即说明 FA 与 NPTA 竞争键合 HSA 的相同位点, 或者可以说 NPTA 在位点 II 位与 HSA 发生作用.

总结以上光谱测定的结果, 得出: NPTA 的存在, 不 仅引起 NPTA-HSA 体系的谱图发生各种变化, 也表明 NPTA 对 HSA 的键合作用使蛋白质的微环境或构象有 所改变.

\subsection{NPTA 与 HSA 键合的物理化学参数}

为进一步定量深入说明 NPTA 对 HSA 的键合情况 及作用机制, 用相关的分析化学公式, 处理光谱法测得 的有关数据, 得到如下表 2 中的物理化学参数 ${ }^{[1]}$. 数据 显示: 不同浓度 NPTA 存在下(约 $4 \sim 40 \mu \mathrm{mol} \cdot \mathrm{L}^{-1}$ 的范 围), 其与 HSA 键合反应的离解常数 $\mathrm{p} K_{\mathrm{a}}$ 呈稍微增大的 趋势, 再次说明 NPTA 与 HSA 有强的键合作用; 而量子 产率 $\Phi$ 值有较大的降低趋势, 表明 NPTA 猝灭 HSA 的 荧光强度较大. 另外, 电荷密度 $\delta$ 值也渐渐减小, 即使 在 NPTA 浓度较低时, 才仅为 0.262 , 也可说明静电作用 不是稳定 NPTA-蛋白体系的一种作用力, 前面所讨论 的键合模式的结果也证实了这点.

表 2 NPTA-HSA 体系一些物理化学参数

Table 2 The parameters of physical properties for NPTA-HSA system

\begin{tabular}{|c|c|c|c|c|c|}
\hline$[\mathrm{NPTA}] /\left(\mu \mathrm{mol} \bullet \mathrm{L}^{-1}\right)$ & 4.36 & 13.10 & 21.84 & 30.56 & 39.30 \\
\hline $\mathrm{p} K_{\mathrm{a}}($ Dissociatin constant $)$ & 7.85 & 7.93 & 8.02 & 8.30 & \\
\hline$\Phi($ Quantum yield $)$ & 0.13 & 0.11 & 0.099 & 0.083 & 0.069 \\
\hline$\delta$ (Charge density) & 0.262 & 0.228 & 0.193 & 0.112 & \\
\hline
\end{tabular}

\section{3 结论}

本文采用光谱法及相关计算机化学的方法, 研究了 一种新合成的三氮唑化合物 NPTA 结构特征及在甲醇 溶液中的光谱特征, 暗示 NPTA 是一种具有开发前景和 实用价值的发光材料. 分子模拟及位点竞争实验确定了 NPTA 在 HSA 上的位点 II 键合; 热力学参数表明 NPTA 键合 HSA 的模式主要为疏水作用; 紫外光谱图及多种 荧光光谱表征了 NPTA 的存在对 HSA 构象的影响. 获 得的不同温度下的键合常数 ( $10^{4}$ 数量级) 说明 NPTA 与 HSA 有较强的键合作用. 并结合 NPTA-HSA 体系的几 种物理化学参数, 从分子水平上了解了 NPTA 与模型蛋 白的键合反应及作用机制.

\section{4 实验部分}

\section{1 仪器与试剂}

RF-5301PC 荧光光度计(日本岛津), 用配套的超级 恒温槽控制实验所需温度, F-7000 菼光光度计(日本日 立), TU-1901 双光束紫外可见光光度计(北京普析通用 仪器有限责任公司), BS124S 电子天平(德国赛多利斯), PH-3S 计(上海精密仪器厂).

三羟甲基氨基甲烷-盐酸缓冲溶液(Tris- $\mathrm{HCl}$ ): 三差弪 甲基氨基甲烷(国药集团化学试剂有限公司, 分析纯), 用二次重蒸水溶解并用盐酸调节 $\mathrm{pH}$ 至 7.40 , 配制成浓 度为 $0.05 \mathrm{~mol} \cdot \mathrm{L}^{-1}$ 的缓冲液. 人血清白蛋白(HSA, 分子 量 66478 , 上海伯奥生物科技有限公司), 用 $\mathrm{pH} 7.40$ Tris- $\mathrm{HCl}$ 缓冲溶液配制, 其储备液 $\left(3.0 \times 10^{-5} \mathrm{~mol} \cdot \mathrm{L}^{-1}\right) 4$ ${ }^{\circ} \mathrm{C}$ 保存于暗处. NPTA 由海南省海南师范大学有机重点 实验室提供(淡黄色粉末，纯度 $>99.9 \%$, 分子量为 271 ), 储备液 $\left(1.3 \times 10^{-3} \mathrm{~mol} \cdot \mathrm{L}^{-1}\right)$, 用甲醇配制. 实验水为二 次蒸馏水; 其他试剂均为分析纯.

\section{2 计算方法}

本文先用 HyperChem 软件获得 NPTA 的最合理分 子结构, 并对各种最合理分子的起始构象进行能量优 化, 以能量最低者(生成热)为药效构象; 再应用 PM3 半 经验方法的 Polak-Ribiere 共轭梯度法进一步优化 NPTA 分子结构, 直到均方根梯度达到 $0.001 \mathrm{kcal} \cdot \mathrm{mol}^{-1[14]}$.

\section{3 分子模拟}

从 Brookhaven 蛋白质数据库中获得 HSA 的晶体结 构(编号为 $1 \mathrm{~h} 9 \mathrm{z}$ ). 在 Silicon Graphics Ocatane2 工作站上 完成计算: 首先根据 Amber 4.0 力场, 用 kollman-allatom 电荷计算出 HSA 三维结构的势能 ${ }^{[15]}$; 再用 Gasteiger-marsili 软件和 tripos 力场优化 HSA 分子的几 何结构, 用 AutoDock3.05 程序来确定 NPTA 与 HSA 分 子之间的相互作用模式，最后用拉马克(LGA)遗传算法 来计算 NPTA 分子与 HSA 结合的可能构象 ${ }^{[16,17]}$. 


\section{4 紫外吸收光谱}

在若干 $10 \mathrm{~mL}$ 比色管中, 依次移取一定量的 HSA 和 NPTA 储备液, 用 $\mathrm{pH} 7.40$ 的 Tris- $\mathrm{HCl}$ 缓冲溶液定容, 以相应试剂的空白为参比, 在紫外可见分光光度计上记 录 NPTA 药物在 200 350 nm 范围内的紫外吸收光谱(1 $\mathrm{cm}$ 石英池). 再以甲醇试剂做参比, 在同样波长范围内, 记录 NPTA 的紫外吸收光谱.

\section{5 二维荧光光谱}

测定在 RF-5301PC (Shimadzu) 苂光光谱仪上进行: 溶液配制方法同上紫外吸收光谱的测定. 选定苂光激发 波长为 $285 \mathrm{~nm}, 1 \mathrm{~cm}$ 石英池, 激发和发射狭缝宽度均为 $5 \mathrm{~nm}$, 测定波长范围在 $290 \sim 500 \mathrm{~nm}$ 的光谱.

\section{6 三维荧光光谱}

测定在 F-7000 (Hitachi)苂光光谱仪上进行：溶液配 制方法同上紫外吸收光谱的测定. 选择荧光激发和发射 狭缝宽度均为 $5 \mathrm{~nm}$ (1 cm 石英池), 分别测定激发波长 范围在 $230 \sim 325 \mathrm{~nm}$, 发射波长 $230 \sim 480 \mathrm{~nm}$ 范围的 HSA 及 HSA-NPTA 溶液体系的三维荧光光谱.

\section{7 荧光偏振}

各向异性 $r$ 的定义如下 ${ }^{[18]}$ :

$$
r=\left(I_{\mathrm{VV}}-G I_{\mathrm{VH}}\right) /\left(I_{\mathrm{VV}}+2 G I_{\mathrm{VH}}\right)
$$

其中, $I_{\mathrm{VV}}$ 和 $I_{\mathrm{VH}}$ 为垂直偏振光激发后的垂直偏振和水平 偏振的发射光强度. $G$ 为仪器校正因子, $G=I_{\mathrm{HV}} / I_{\mathrm{HH}}, I_{\mathrm{HV}}$ 和 $I_{\mathrm{HH}}$ 为水平偏振光激发下的垂直偏振和水平偏振光的 发射光强度. 激发与发射波的狭缝宽均为 $5 \mathrm{~nm}$.

\section{8 固定波长同步荧光测定}

以 230 为激发波长, 290 或 $245 \mathrm{~nm}$ 为发射波长 $(\Delta \lambda$ $=60 \mathrm{~nm}$, 或 $\Delta \lambda=15 \mathrm{~nm})$, 测定加入不同浓度 NPTA 后 HSA-NPTA 溶液体系的同步苂光光谱(激发和发射狭缝 宽度均为 $5 \mathrm{~nm}$ ).

\section{9 键合常数的测定}

配制不同浓度比的 HSA-NPTA 溶液体系, 选择激 发波长和发射波长分别为 285 和 $348 \mathrm{~nm}$, 测定三种不同 温度 $\left(26,36,46{ }^{\circ} \mathrm{C}\right)$ 下的菼光强度, 再依据相应的公式
计算结合常数 ${ }^{[9]}$.

\subsection{0 位点标记竞争实验}

固定蛋白的浓度，向 NPTA-HSA 体系分别加入一 定量的竞争试剂 PB、FA 和 Dig, 分别作为位点 I、位点 II、位点 III 位的标记药物 ${ }^{[13]}$. 选择激发波长和发射波长 分别为 285 和 $348 \mathrm{~nm}$, 测定 HSA-NPTA 体系在 $26{ }^{\circ} \mathrm{C}$ 的 苂光强度.

\section{References}

[1] Wu, L. Y.; Chen, Y. X.; Tang, M.; Song, X. M.; Chen, G. Y.; Song, X. P.; Lin, Q. Synlett 2012, 1529.

[2] Shi, J. G.; Yang, X. Y.; Xu, L. Z. J. Qingdao Univ. Sci. Technol. 2003，24，309. (时建刚，杨秀英，许良忠，青岛科技大学学报， 2003, 24, 309.)

[3] Luo, Y.; Chen, T. F.; Huang, X. C.; Wang, Y.; Wong, Y. S.; Zheng, W. J. Acta Chim. Sinica 2012, 70, 1295. (罗懿, 陈填烽, 黄晓纯, 王式, 黄荫成, 郑文杰, 化学学报, 2012, 70, 1295.)

[4] Ma, G. C.; Tang, R. H.; Zhen, S. C. The Methods and Application of Pharmaceutical Analysis, Science Press, Beijing, 2001, pp. 210 218. (马广慈, 唐任睘, 郑斯成, 药物分析方法与应用, 科学出版 社, 北京, 2001, pp. 210 218.)

[5] Xu, J. G.; Wang, Z. B. Fluorescence Analysis (Third Edition), Science Press, Beijing, 2006, pp. 22 26. (许金钩, 王尊本, 荧光分析 法(第三版), 科学出版社, 北京, 2006, pp. 22 26.)

[6] Sudlow, G.; Birkett, D. J.; Wade, D. N. Mol. Pharmacol. 1976, 12, 1052.

[7] Wolfbeis, O. S.; Leiner, M.; Hochmuth, P.; Geiger, H.; Bunsenges, B. Bunsenges. Phys. Chem. 1984, 88, 759.

[8] Valeur, B. Molecular Fluorescence: Principles and Applications, Wiley-VCH Verlag GmbH. ISBNs: 3-527-29919-X (Hardcover), 3-527-60024-8 (Electronic), 2001, pp. 56 68 .

[9] Wei, X. F.; Liu, H. Z. Chin. J. Anal. Chem. 2000, 28, 699. (魏晓芳, 刘会洲, 分析化学, 2000, 28, 699.)

[10] Yang, P.; Gao, F. Principles of Bioinorganic Chemistry, Beijing, Science Press, 2002, pp. 325 331. (杨频, 高飞, 生物无机化学原 理, 科学出版社, 北京, 2002, pp. $325 \sim 331$.)

[11] Mohammed, H. R.; Toru, M.; Tomoko, O.; Keishi, Y.; Masaki, O. Biochem. Pharm. 1993, 46, 1721.

[12] Ross, P. D.; Subramanian, S. Biochem. 1981, 20, 3096.

[13] Carter, D. C.; He, X. M.; Munson, S. H.; Twigg, P. D.; Gernert, K. M.; Broom, M. B.; Miller, T. Y. Science 1989, 244, 1195.

[14] HyperChem 6.01. Hypercube, Inc., 2000.

[15] SYBYL Software, Version 6.9, St. Louis, Tripos Associates Inc, 2002.

[16] Morris, G. M.; Goodsell, D. S.; Huey, R.; Olson, A. J. J. Comput-Aided. Mol. Des. 1996, 10, 293.

[17] Morris, G. M.; Goodsell, D. S.; Halliday, R. S.; Huey, R.; Hart, W. E.; Belew, R. K.; Olson, A. J. J. Comput. Chem. 1998, 19, 1639.

[18] Lakowicz, J. R. Principles of Fluorescence Spectroscopy, Plenum, New York, 1983, pp. 341 379 . 